\title{
Efficacy of a Treatment for Gonarthrosis Based on the Sequential Intra-Articular Injection of Linear and Cross-Linked Hyaluronic Acids
}

\author{
E. Barbieri ${ }^{1,2}$, I. Capparucci ${ }^{1}$, F. Mannello1, G. Annibalini ${ }^{1}$, S. Contarelli', L. Vallorani', \\ A. M. Gioacchini ${ }^{1}$, D. Ligi ${ }^{1}$, R. Maniscalco ${ }^{1}$, M. Gervasi ${ }^{1}$, T. Tran Dang Xuan ${ }^{3}$, \\ C. Bartolucci ${ }^{1}$, V. Stocchi ${ }^{1}$, P. Sestili ${ }^{1}$ \\ 1 Department of Biomolecular Sciences, University Urbino Carlo Bo, via A. Saffi 2, 61029 Urbino, Italy \\ 2 Interuniversity Institute of Myology \\ 3 Stem Cells Center, Van Hanh Hospital, Hochiminh City, Vietnam
}

\section{CORRESPONDING AUTHOR:}

\section{Elena Barbieri}

Department of Biomolecular Sciences

University Urbino Carlo Bo

Via A. Saffi 2

61029 Urbino, Italy

Phone: +390722 303417

E-mail: elena.barbieri@uniurb.it

\section{DOI:}

10.32098/mltj.04.2019.17

LEVEL OF EVIDENCE: 4

\begin{abstract}
SUMMARY
Background. This study evaluates the clinical and biochemical effects of sequential intra-articular (IA) injections of linear (LHA) and cross-linked hyaluronic acid (CLHA) in patients with Gonarthrosis (GA).

Methods. Thirty-nine (39) patients (age $64.89 \pm 8.83$ ) received first the LHA injection $(0.8-1.2 \mathrm{MDa}, 32 \mathrm{mg} / 2 \mathrm{ml})$ and after 1 week the CLHA (1.0 MDa and $2.0 \mathrm{MDa}, 75 \mathrm{mg} / 3 \mathrm{ml})$ one; this round was repeated after 6 months. Clinical assessments - i.e. ultrasonography, visual analogic scale (VAS) for pain, range of motion (ROM) and Western Ontario and McMaster Universities Osteoarthritis (WOMAC) index - were performed at baseline and at specific time points up to 12 months. Relevant markers were determined in blood and synovial fluid (SF) at selected intervals. SF from patients with recurrent effusion was subjected to proteomic analysis.

Results. This schedule improved joint pain and function, and promoted a reduction of inflammatory cytokines (IL-1 $\beta$, IL-9 and IL-17) in plasma and SF; cartilage thickness increased at 12 months and the increase negatively correlated with the baseline levels of C-telopeptide of type II collagen (CTX-II). SF proteomic revealed that proteins associated with inflammation (Apolipoprotein A-1, $\alpha-1$ antitrypsin and $\operatorname{IgK}$ ) decreased, while the IL-1 inhibitor Transthyretin increased.

Conclusions. This schedule represents an effective treatment whose benefits persist up to 12 months after baseline.
\end{abstract}

KEY WORDS

Cytokines; CTX-II; gonarthrosis; byaluronic acid

\section{BACKGROUND}

Gonarthrosis (GA) is a leading cause of disabling joint disease, especially in the elderly (1): the increase in life expectancy and the aging of the population will make arthropathy the fourth leading cause of disability in 2020 with the ensuing socio-economic consequences $(1,2)$. Arthropathy was observed to have a $104.9 \%$ rise in disability-adjusted life-years index (or $8.8 \%$ when age-standardized) from 1990 to 2016 (2).

Intra-articular (IA) injections provide a non-operative strategy for GA management (3). Currently, the best evidence suggests that hyaluronic acid (HA) is an effective intervention in treating GA without increased risk of adverse events. Recently, systematic reviews of overlapping meta-analysis and 'real-life' studies demonstrated the effectiveness of IA HA injections (4).

Biologically, HA is not an inert molecule. The native HA appears with a high molecular weight (MW) that can be degraded into smaller fragments due to glycosidase activity induced by several physio-pathological adaptive responses (2). Different HA size fractions have been evaluated for several biomedical applications, in particular for GA viscos- 
upplementation. However, the heterogeneity of the studies in comparing low-MW HA ( 80-800 KDa) and high-MW HA ( 2.0 MDa) for treating knee injury, limits definitive conclusions. Indeed, the HA fragments, depending on their size, display different rheological properties and have been shown to either stimulate or inhibit inflammatory response in targeted cells and in diseased tissues, and to differently modulate the production of specific inflammatory mediators $(5,6)$. In agreement with the notion of the size- and structure- dependent bioactivity of HA, hybrid preparations of low- and high-MW HA $(7,8)$, crosslinked-HA (9) and hexadecylic-derivatized HA (10) show even further variations/ complexity in terms of pharmacodynamics as compared to the native, linear polymers.

From the clinical point of view, according to a Cochrane Database Systematic Review analysis based on a significant number of controlled trials, it has been demonstrated that HA injections significantly reduce pain in knee osteoarthritis (11).

Injectable preparations for IA viscosupplementation consist of linear (LHA) or cross-linked (CLHA) commercially available HA, this latter reputed to be generally more resistant to enzymatic degradation (9).

The effect of HA in knee arthropathy has often been attributed to its biomechanical and viscosupplementation properties; however there is growing evidence suggesting that the benefits of HA depend also on other biological actions on both inflammation and/or cartilage degradation $(5,12)$.

The aim of this study was to evaluate the clinical and biochemical effects of timed-sequential IA injections of LHA and CLHA formulations in ameliorating joint pain and function in patients with knee GA also focusing on the modulation of cartilage degradation markers, and of cytokines implicated in GA pathogenesis (13).

\section{MATERIALS AND METHODS}

\section{Study design}

We performed a descriptive longitudinal study, with followup visits up to 12 months. Patients with symptomatic mildmoderate GA, grades II-III according to Kellgren-Lawrence (KL) score radiographically examined no longer than three months before the beginning of the study, were enrolled.

Exclusion criteria were the following: joint infection, inflammatory joint disease, osteonecrosis, positive synovial fluid culture, reduced range of motion, large knee circumference $(>45 \mathrm{~cm})$, recent IA HA injections and knee trauma or surgery, full-thickness cartilage loss in index knee and/or treatments with steroids or non-steroidal anti-inflammatory drugs within the previous 3 months (acetaminophen was only allowed), rheumatic pathologies, endocrinopathies, malignancies and systemic diseases.

The study meets the ethical standards of the journal. In particular, all experimental procedures were carried out according to the principles and recommendations described elsewhere (14). After approval from the institutional ethical committee on November $15^{\text {th }} 2016$, the study was carried out according to Helsinki Declaration for research with human volunteers (1975) and all patients signed an informed consent form to participate.

Thirty-nine (39) patients meeting the inclusion criteria were enrolled in the study. Anthropometric data (age, sex, height, weight, and body mass index, body mass index, BMI) and level of physical activity (PA) measured by the Saltin-Grimby Physical Activity Level Scale (15) were recorded at baseline (Table I). Clinical and functional assessments were performed during the sequential IA injections of LHA and CLHA (baseline/1-Wk and 6 months/1-Wk) and repeated each 3 months up to 12 months as described in the study design in Figure 1.

\section{HA treatments}

Viscosupplementation regimen consisted in two sequential IA injections of different HA formulation: the first consisted of 0.8-1.2 MDa LHA (32mg/2ml, RegenFlex Starter) and the second after 1 week containing three different CL fractions of $\mathrm{HA}, 1.0 \mathrm{MDa}$ and 2.0 MDa, intercalated with a

Table I. Study group characteristics.

\begin{tabular}{lll}
\hline Variable & & \\
\hline Subjects & 39 & \pm 8.8 \\
\hline Age (years) & 64.9 & \\
\hline Male & 26.0 & \\
\hline Female & 13.0 & \\
\hline Smoking & 16 & \pm 6.3 \\
\hline (yes or past) & & \pm 0.1 \\
\hline Weight $(\mathrm{kg})$ & 69.9 & \pm 2.4 \\
\hline Height $(\mathrm{m})$ & 1.7 & \pm 0.2 \\
\hline BMI $\left(\mathrm{kg} / \mathrm{m}^{2}\right)$ & 23.8 & \\
\hline PA level & 1.6 & \\
\hline Bilateral $(\mathrm{Yes})$ & 14 & \\
\hline Bilateral $(\mathrm{No})$ & 25 & \\
\hline SF & 19 & \\
\hline DRY & 24 & \\
\hline BMI, body mass & & \\
\hline
\end{tabular}

BMI, body mass index; $P A$, physical activity.

SF, synovial fluid; DRY, no synovial fluid.

Values are the mean $\pm S E$. 


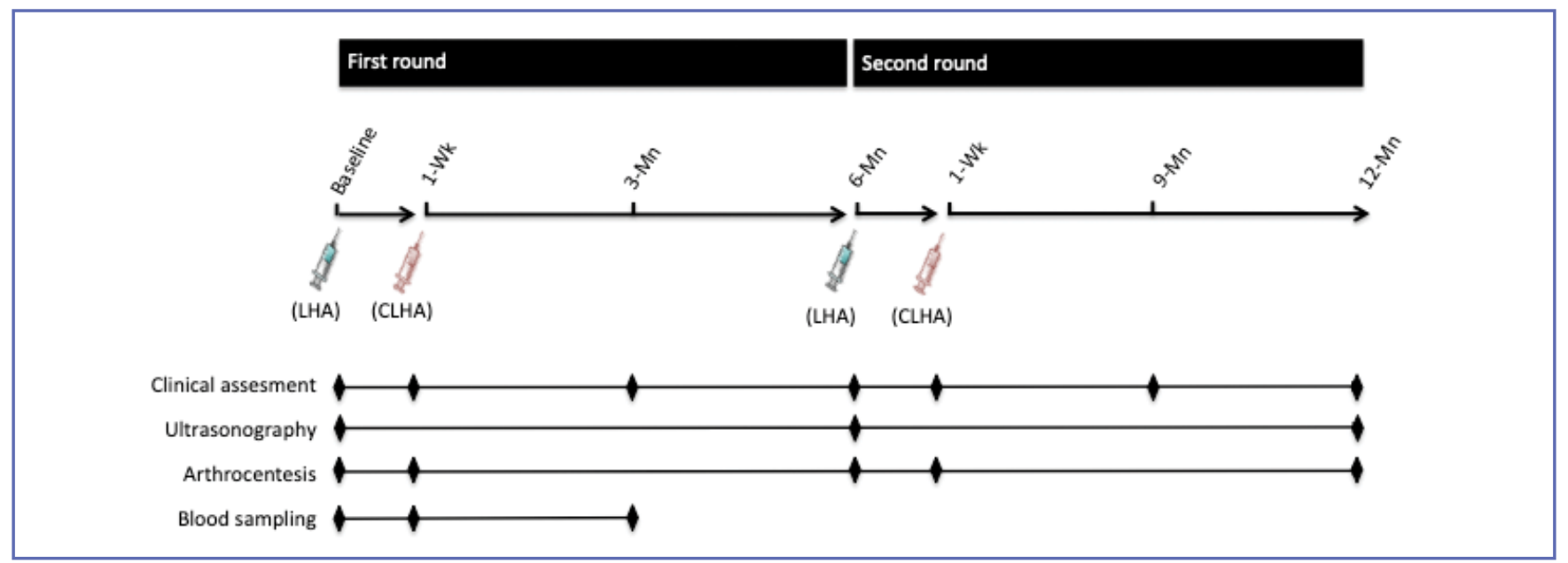

Figure 1. Design of the study procedures. The sequential IA injections of HA in GA patients consisted of the first injection with LHA followed by a second of CLHA after 1 week. The same round was repeated after 6 months from the baseline. The timelines of clinical assessments, ultrasonograpy, arthrocentesis, and blood sampling are also shown.

LHA fraction of $0.5 \mathrm{MDa}$, this latter representing $10 \%$ of the total HA amount $(75 \mathrm{mg} / 3 \mathrm{ml}$, RegenFlex Bioplus).

The knee joint injections were performed under sterile conditions by inserting a 21 -gauge needle into the patellofemoral joint space by super lateral approach while the patients were in a supine position as described in (16). The identical treatment scheme with the two sequential IA injections was repeated after 6 months from baseline (Figure 1); the same physician performed all the injections. Synovial fluid (SF) was diagnosed by clinical evaluation and, when present, it was withdrawn before each HA injection. The number of arthrocenteses and the amounts of fluid aspirated were registered.

\section{Clinical and functional assessments}

Functional parameters included pain intensity and range of motion (ROM). Pain intensity was determined using a visual analogue scale (VAS) ranging from 0 to 10 ; ROM (knee flexion and extension) was goniometrically-assessed according to (17); Western Ontario and McMaster Universities Osteoarthritis (WOMAC) index was used for knee functional limitation. All WOMAC data presented in this report have been normalized using average score on 0 to 10 scales, similarly to other studies (18). Satisfaction of patients was also evaluated using the Likert Scale (19).

\section{Ultrasonographic examination}

Mean cartilage thickness was measured by means of ultrasound imaging (Esaote MyLab ${ }^{\mathrm{TM}} 70$ XVG ultrasound machines equipped with $12 \mathrm{MHz}$ and $18 \mathrm{MHz}$ linear transducers). First, the intercondylar notch area, including femoral condyles beyond patellar bone, was depicted. Successively, the cartilage, in medial and lateral femoral condyles, as well as intercondylar (sulcus) was scanned by sweeping the full surfaces from proximal to distal, using the probe always in transverse position. The ultrasound beam was held perpendicular respect the femur surface (20). The knees were flexed maximally in order to make a great part of the weight-bearing surface of the femoral condyles accessible to ultrasound assessment. The grade of knee flexion ranged ca. 100-140 . The sonographer (CB), who has over 30 year experience in musculoskeletal sonography, was blinded to the history, clinical findings and imaging data of the patients.

\section{Biochemical assessment}

Plasma samples were collected at baseline, after 1 week and 3 months from the first treatment and analysed for CTX-II and cytokine content. When available (see Results section), SF samples (with the exception of those positive for haemolysis) were collected before $\mathrm{HA}$ injections along the study. Samples were maintained at $-80^{\circ} \mathrm{C}$ until analysis (Figure 1). Enzyme-linked immunosorbent assay (ELISA) test kits were used to determine the plasma and SF levels of IL-1 $\beta$, IL -17 and IL-9 (BMS224HS High Sensitivity, BMS2017, BMS2081 ELISA ThermoFisher Scientific, SrL) and CTX-II (ELISA CTX-II MBS261323 Novus Biologicals, SrL). Human lubricin (ELISA LS-F7095 LifeSpan, Inc.) elastase (ELISA NBP2-60501 Novus Biologicals, SrL) and CTX-II in SF were also quantified. 


\section{Synovial fluid proteomic analysis}

Aliquots of $1 \mathrm{ml}$ of the SF from patients characterized by chronic synovitis and recurrent knee effusion collected at baseline, at 6 and at 12 months were pooled; the protein concentration of each SF pool was determined according to Bradford's method to define the volume of sample to be loaded in two-dimensional electrophoresis (2-DE). 2 -DE was carried out as previously described in Sestili et al. (21).

\section{Statistical analysis}

Descriptive statistics indices i.e. mean and standard deviations were established for the analysed parameters. Separate one-way repeated measure ANOVAs were performed on VAS, knee ROM, WOMAC and plasma cytokine levels. The Bonferroni post-hoc test was used for post-hoc analysis. Paired $t$-tests were used to compare SF parameters between the baseline and 1 week post HA treatment. Pearson correlation was used to analyse the relationship between baseline CTX-II concentration and changes of cartilage thickness at 12 months. The level of statistical significance was set at $p<0.05$. Statistical analyses were performed with SPSS (IBM SPSS Statistics for Windows v20.0, IBM Corp.).

\section{RESULTS}

Thirty-nine (39) patients were selected for the study based on the inclusion criteria detailed above. The distribution of gender, age, weight, smokers, BMI, level of PA, and KL grade is described in Table $\mathbf{I}$. The 14 patients suffering of bilateral GA received injections in both the knees. No dropout and no local/systemic reactions or side effects were noted.

As shown in Table II, VAS score decreased immediately after the first LHA injection and remained low over 12 -month follow-up $(p<0.05)$. The analysis of the variation of VAS scores over time shows more pronounced drops corresponding with the two injections of LHA; the sequential injections of CLHA seemed to exert a long lasting stabilization of these results. Importantly, ROM, VAS and WOMAC recorded at the 6 months plus 1 -week point and later ones, showed a significant amelioration as compared to the data obtained not only at baseline, but also to those at 6 months (Table II).

On the whole patients experienced significant improvements in total WOMAC scores, as well as in ROM values, although compared to VAS reduction. WOMAC and ROM seemed to change more gradually over time.

Arthrocentesis was performed in 19 patients at baseline in the presence of synovial effusion (Table II). The volume

Table II. Clinical results at baseline and follow-up visits.

\begin{tabular}{|c|c|c|c|c|c|c|c|}
\hline & Baseline & $1-\mathrm{Wk}$ & 3-Mn & 6-Mn & 6-Mn+1Wk & 9-Mn & 12-Wk \\
\hline VAS & $11.27 \pm 15.32$ & $\begin{array}{l}8.16 \pm \\
17.25 \%\end{array}$ & $8.86 \pm 17.49^{*}$ & $8.99 \pm 17.52 *$ & $\begin{array}{l}7.33 \pm \\
18.54 * 5\end{array}$ & $\begin{array}{l}7.57 \pm \\
18.95 * s\end{array}$ & $\begin{array}{l}7.68 \pm \\
19.03 * 8\end{array}$ \\
\hline WOMAC & $4.84 \pm 1.08$ & $6.02 \pm 0.76^{*}$ & $7.00 \pm 0.85^{*}$ & $\begin{array}{l}7.00 \pm \\
0.85 *\end{array}$ & $\begin{array}{l}7.86 \pm \\
0.71 * s\end{array}$ & $\begin{array}{l}7.96 \pm \\
0.64 * s\end{array}$ & $8.02 \pm 0.65 *$ \\
\hline
\end{tabular}

\begin{tabular}{|c|c|c|c|c|c|c|c|}
\hline \multicolumn{8}{|l|}{ Arthrocentesis } \\
\hline Number $(N)$ & 19 & 8 & & 8 & 8 & & 8 \\
\hline Volume $(c c)$ & $20.80 \pm 4.67$ & $19.10 \pm 3.65$ & & $12.00 \pm 3.92 *$ & $7.60 \pm 3.13 * s$ & & $6.60 \pm 2.85 *$ \\
\hline \multicolumn{8}{|l|}{ Satisfaction } \\
\hline Not at all & & $1(2.56 \%)$ & 0 & 0 & 0 & 0 & 0 \\
\hline Slightly & & $34(87.18 \%)$ & $12(30.77 \%)$ & $12(30.77 \%)$ & $6(15.38 \%)$ & $2(5.13 \%)$ & $2(5.13 \%)$ \\
\hline Very & & 0 & $5(12.82 \%)$ & $5(12.82 \%)$ & $6(15.38 \%)$ & $10(25.64 \%)$ & $10(25.64 \%)$ \\
\hline Extremely & & 0 & $1(2.56 \%)$ & $1(2.56 \%)$ & $3(7.69 \%)$ & $4(10.26 \%)$ & $4(10.26 \%)$ \\
\hline
\end{tabular}

VAS, Visual analogue scale; ROM, Range of motion (degree); WOMAC, Western Ontario and McMaster Universities Osteoarthritis (functional subscale, normalized using average score on 0 to 10 scales).

Arthrocenteses were performed at the indicated time points and the volume of the resulting SFs was determined immediately before each $H A$ injection and at 12 months (means \pm SE).

* Significantly different from baseline $(p<0.05)$. "Significantly different from 6 months $(p<0.05)$. 
and/or the occurrence of SF were significantly reduced after 6 and 12 months. All samples had clear appearance and high viscosity. In the microscopic evaluation, the white blood cell contained $40 \%$ of polymorphonuclear leukocytes ruling out infection as the cause of symptoms (data not showed).

All the above results are grounded by patients' satisfaction report, which was evaluated by means of a five-points Likert scale: indeed at 12 months, 37 patients were somewhat/very/extremely-satisfied of the treatments received (Table II).

The ultrasound images taken at baseline and at 12 months follow-up were used to monitor the femoral cartilage thickness (Figures 2A-F). Three patients' lateral femoral knees cartilage ultrasonographs, representative of the clinical scenario of this study, are shown in Figures 2A-F. The first case (Figure 2A-B) refers to the patient who experienced the most consistent amelioration of cartilage condition observed in the study; the second (Figure 2C-D) is repre-

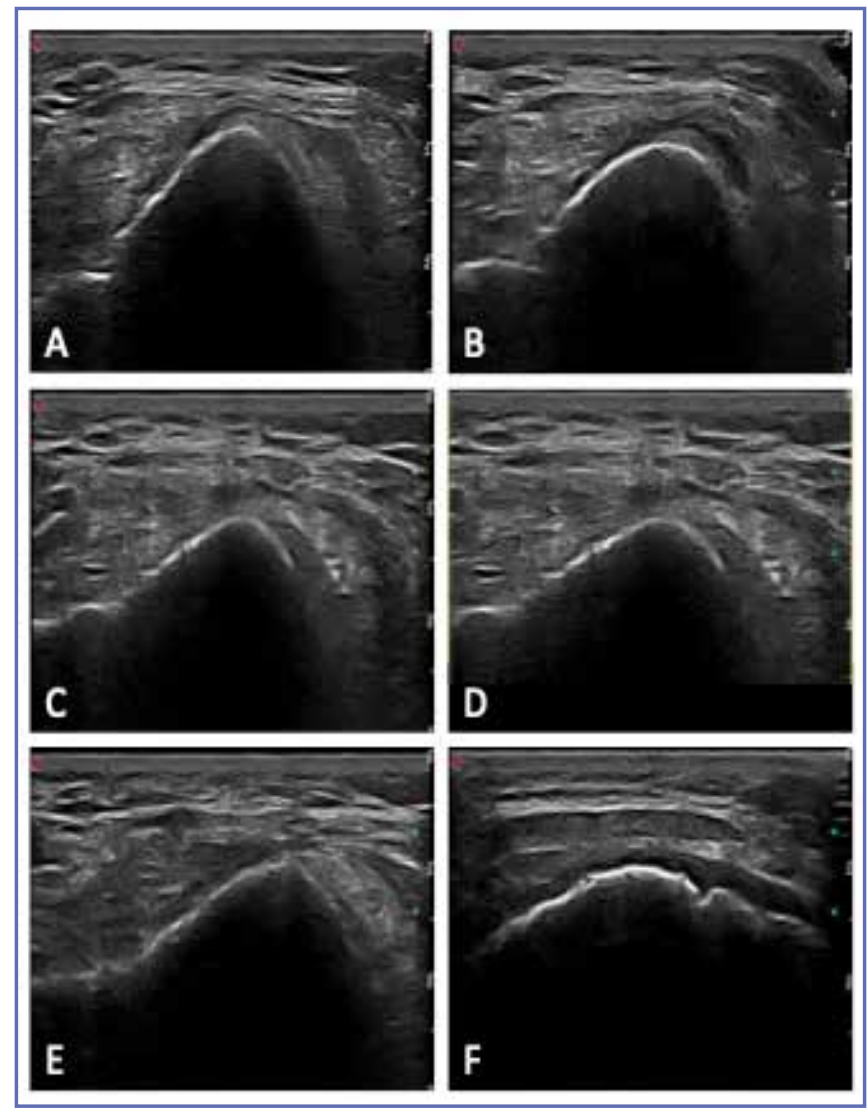

Figure 2. Representative ultrasonographs of lateral femoral cartilage changes. Ultrasonographs of the lateral femoral cartilage from three patients at baseline and 12 months follow-up are shown in A-B, C-D and E-F, respectively. sentative of the average/intermediate clinical conditions, i.e. moderate symptomatic conditions; the third case (Figure 2E-F) refers to an elderly patient with a severe symptomatic condition characterized by a discontinuous aspect of the cartilage surface and chronic synovitis. Notwithstanding the clinical differences, these three patients showed ultrasonographic signs of improvement of cartilage conditions and thickness at the 12 months follow-up, although to different extents. On the whole, at 12 months the mean cartilage thickness of the knee in the enrolled patients was significantly greater than at baseline $(0.84 \pm 0.19 \mathrm{~mm}$ and 2.72 $\pm 0.86 \mathrm{~mm}$, respectively; $p<0.05$ ). CTX-II plasma concentration did not change significantly after 3 months from the baseline $(0.69 \pm 0.28 \mathrm{ng} / \mathrm{ml}$ and $0.70 \pm 0.24 \mathrm{ng} / \mathrm{ml}$, respectively; $p>0.05)$. Pearson correlation analysis was run to assess the relationship between baseline CTX-II plasma level and cartilage thickness changes at 12 months: a statistically significant negative correlation between these two parameters $(r=-0.517 ; p<0.05)$ was found.

\section{Plasma and synovial inflammatory markers}

The plasma inflammatory markers IL- $1 \beta$ and IL-9 were significantly reduced after 3 months $(p<0.05)$, while IL-17 was slightly and non-significantly reduced at 1 week and 3 months (Figure 3A, B, C).

Synovial IL-1 $\beta$, IL-9 and IL-17 levels (Figures 3D, 3E and 3 F) decreased at 1 week after baseline $(p<0.05)$, while those of the cartilage markers CTX-II, lubricin and elastase (Figures 3G, 3H, 3I) did not significantly change.

In a subgroup of 8 patients with persistent SF effusion, we also performed a proteome analysis of SF at baseline, 6 and 12 months (Figures 4A-C). The identification of the protein differentially expressed in the course of the study revealed a decrease of inflammation-related proteins Apolipoprotein A-1, $\alpha-1$ antitrypsin and $\operatorname{IgK}$ chain, along with an increase of Transthyretin, an inhibitor of IL-1 $\beta$.

\section{DISCUSSION}

The present study assessed the clinical outcomes of a therapeutic course consisting in the timed and sequential cycles of LHA and CLHA IA injections repeated after 6 months for the treatment of KL II and III grade GA over a 12 months follow up period, its effect on cytokine profile and cartilage degradation markers in plasma and, when available, in SF. Our data indicate that this therapeutic regimen promoted a significant, rapid and durable amelioration of GA in all the enrolled patients. Clinical, functional and most of the biochemical markers considered (from plasma and SF) improved at 6 and in some cases also at 12 months. Relief 


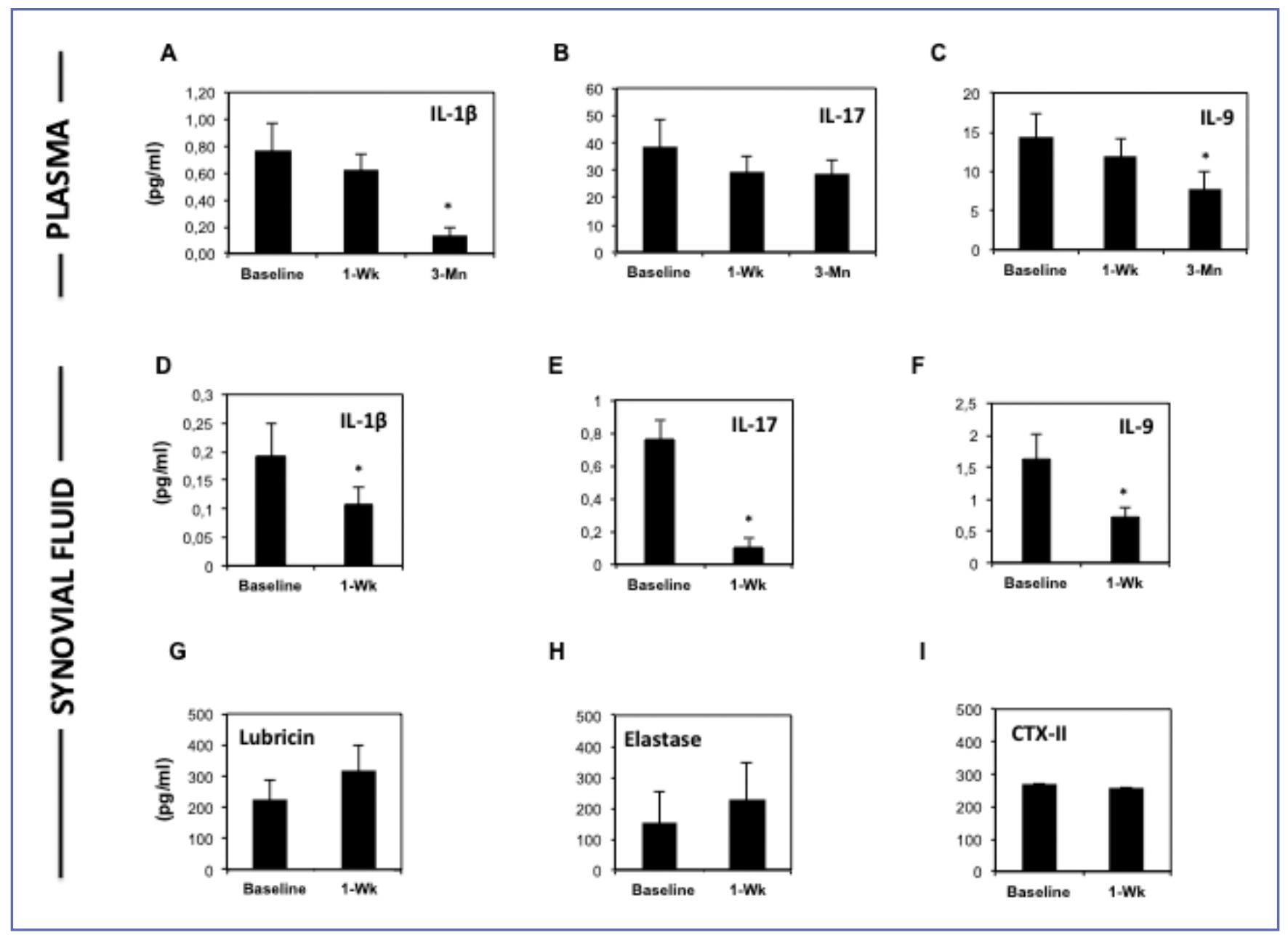

Figure 3. Plasma and synovial levels of relevant biochemical markers of inflammation and cartilage degradation. Plasma IL-1 $\beta$, IL-9, IL-17 levels were determined at baseline, 1 week and 3 months. Synovial IL-1 $\beta$, IL-9, IL-17, lubricin, elastase and CTX-II levels were determined at baseline (immediately before the first injection with LHA) and 1 week. Values are means \pm SE. *, significantly different as compared to baseline $(p<0.05)$.

from invalidating symptoms was very rapid and continued to increase - or at least did not reverse - over time: indeed better VAS, ROM and WOMAC scores (and SF volume reduction in recurrent knee effusion cases), were observed immediately after the first round of HA injections and up to the 6 months follow-up. The second round of HA injections resulted in a further amelioration of VAS, ROM and WOMAC; most of the effects were afforded by LHA injections, while CLHA ones, from their side, seemed to stabilize and/or slightly improve the results attained with the LHA injections.

Accordingly, all the biochemical and clinical outcomes (namely satisfaction of patients and SF volume, where available) invariably tended to ameliorate at the selected checkpoints.
Intra-articular administration of HA got the consensus for the conservative treatment of GA $(3,22)$. In line with this notion, here we observed that HA not only attenuated the nociceptive response in arthrosic joints, but also seemed to positively affect cartilage condition. This notion is supported by the outcomes of ultrasonographic observations at 12 months $v$ s baseline. The use of ultrasound assessment of articular cartilage is in accordance with previous investigations, where ultrasound examination provided direct information on soft tissue degeneration supplying relevant additional diagnostic information on cartilage-specific morphological changes $(23,24)$. Ultrasound imaging appear to be useful to assess relative cartilage thickness in the middle and posterior medial femoral regions as done in our study, and it 


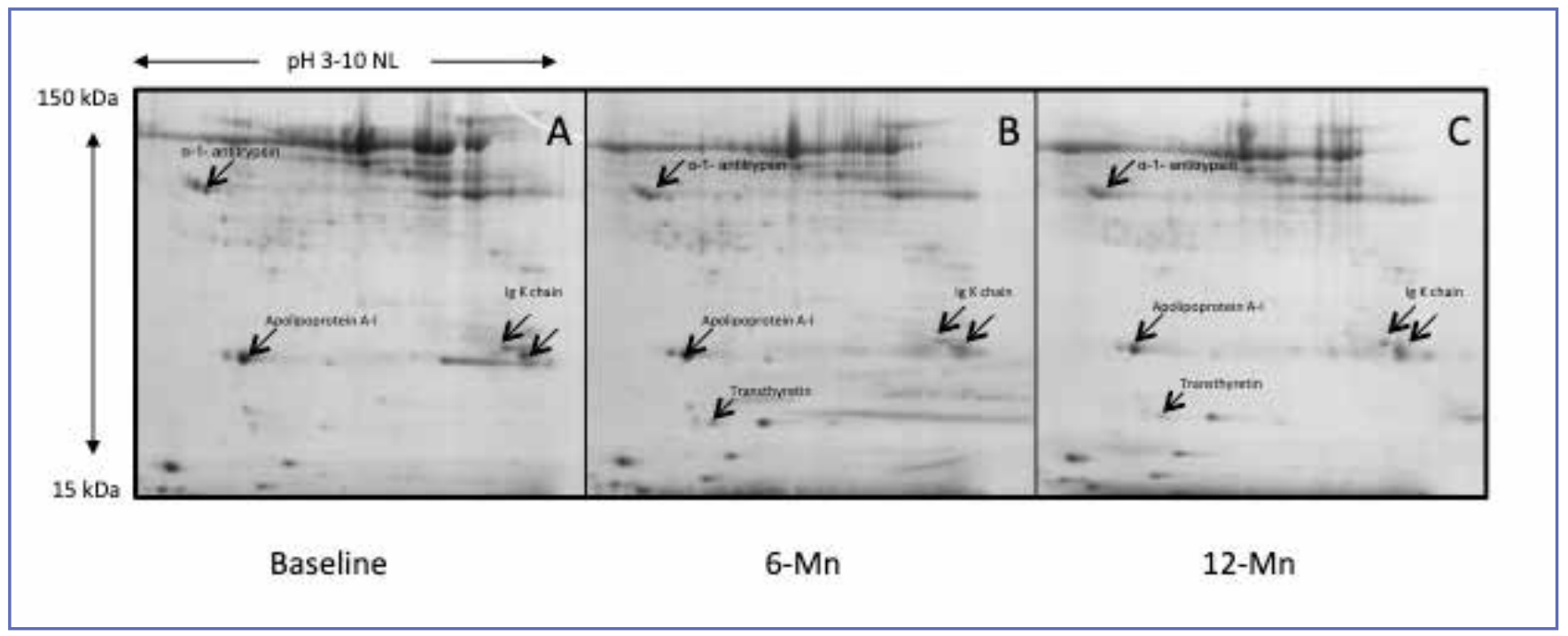

Figure 4. Proteomic analysis of synovial fluid. Representative 2D electrophoreses of the SF from a subgroup of patients with severe synovitis at baseline (A), after 6 months (B) and 12 months (C). Arrows refer to relevant proteins, which are differently expressed over time.

has also been found in good agreement with the measures obtained by magnetic resonance imaging by several authors $(25,26)$. The ameliorative cartilage conditions observed at 12 months $v$ s baseline were supported, from a biochemical perspective, by the lack of plasma CTX-II increase at three months. Indeed, Bruyere et al. reported that an increase of CTX-II over this period ( 3 months) is predictive of cartilage loss at later times (i.e.12 months) (27). Moreover from this study it is also clear the efficacy of HA in modulating specific pro-inflammatory mediators. Indeed, relevant circulating markers of inflammation associated to GA, namely IL- $1 \beta$ and IL-9, decreased at 3 months. For the sake of completeness, although IL-9 is considered as a pleiotropic multifunctional cytokine - whose role depends on the microenvironment and specific condition settings - in joint and rheumatic diseases it mostly acts as a pro-inflammatory cytokine (28). Reduction of IL-1 $\beta$, IL-9 and IL-17 levels was also found in the SFs from patients at 1 week after LHA treatment.

In line with the biochemical data described so far, the proteomic analysis of SFs at 6 months and 12 months revealed a HA-induced modulation of specific proteins involved in the inflammatory response: in particular the levels of Apolipoprotein A-1, IgK chain and $\alpha$-1-anti-trypsin decreased over time, while Transthyretin increased. Apolipoprotein A-1 is a high-density lipoprotein playing a key role in the transport and delivery of lipids which also possesses pro-inflammatory properties: indeed it has been shown to promote the induction of strong matrix metalloproteinases expression and to exert a pro-inflammatory effect within SF (29); the IgK chain is involved in antibody production, and increased levels of its free light chain can be detected in inflamed sites (30); $\alpha$-1-anti-trypsin increases in the course of active progression of cartilage decay and of inflammation (31); finally transthyretin, which has an anti-inflammatory activity, is an inhibitor of constitutive IL- $1 \beta$ production and is able to chelate amyloid preventing its deposition in knee joint (32).

On the whole, the biochemical data indicate that the HA-treatment paradigm adopted in this study, along with the mechanical benefit due to viscosupplementation, promotes an attenuation of inflammatory markers, which turns, at 12 months, into the overall amelioration of kneejoints as assessed ultrasonographically as well as into the clinical and functional improvement, namely pain, ROM and WOMAC and patients' satisfaction scale.

As previously noted the clinical and functional responses described herein indicate a rapid and early clinical efficacy phase ( 1 week) coincident with- and causally related to- the first injection of LHA, followed by a general progressive and more gradual amelioration in the periods following CLHA injection. Indeed the first articular viscosupplementation of LHA promoted a prompt and dramatic pain reduction invariably perceived by patients associated with an overall anti-inflammatory tendency (as indicated by plasma and SF cytokine and biochemical markers trends).

Although it is a merely tempting hypothesis, it could be speculated that LHA promotes a rapid pre-conditioning of joint environment, establishing a favourable ground for the 
subsequent injection (post 1 week) of CLHA. Notably HA degradation rate - paralleling to the loss of its activity - is very high in inflamed joints (33). Hence, it is conceivable that a pre-conditioned, less inflamed joint environment, might contribute to further reduce the per se slow degradation rate of subsequently injected CLHA, further prolonging its activity. Indeed HA crosslinking reduces HA sensitivity to hyaluronidase-mediated degradation rendering CLHA more stable as compared to LHA (9). Such an "extra prolonged" activity would likely turn into a more consistent joint clinical improvement. It is worth noting that such an hypothesis is in keeping with the significant reduction of IL-1 $\beta$ and IL-9 still observed at 3 months after the first round of injections, and with clinical, functional and ultrasonographic outcomes at the 12 months follow-up.

Apart from the biochemical issues, established observations indicate that in symptomatic mild-moderate GA patients $\mathrm{HA}$, due to its role in the joint lubrication, may reduce the joint friction coefficient that is the main risk factor for degenerative joint pathologies and pain $(3,4)$. Again, CLHA, being more resistant to hyaluronidase degradation, affords a prolonged visco-supplementation and lubricant effect, which, under the specific schedule adopted herein, might be even longer.

On the whole, despite some limitations of this report that include the lack of a control group and the variability of the basal levels of the inflammation markers - common in similar studies trying to focus on "real life" situations - combining the clinical and biochemical results of this descriptive study, we show that timed-sequenced IA injections of LHA and CLHA represents a safe and highly

\section{REFERENCES}

1. Briggs AM, Woolf AD, Dreinhöfer K, Homb N, Hoy DG, Kopansky-Giles D, et al. (2018) Reducing the global burden of musculoskeletal conditions. Bulletin of the World Health Organization. 96(5), 366-8.

2. Necas J, Bartosikova L, Brauner P, Kolar J. (2008) Hyaluronic acid (hyaluronan): a review. Veterinarni medicina. 53(8), 397-411.

3. Maheu E, Bannuru RR, Herrero-Beaumont G, Allali F, Bard H, Migliore A. (2018) Why we should definitely include intra-articular hyaluronic acid as a therapeutic option in the management of knee osteoarthritis: Results of an extensive critical literature review. Seminars in Arthritis and Rheumatism.

4. Migliore A, Frediani B, Gigliucci G, Anichini S, Cassol M, Crimaldi S, et al. (2018) One-year follow-up showing effects of single intra-articular injection of hyaluronic acid (1,500-2,000 $\mathrm{kDa}$ ) in symptomatic knee osteoarthritis. Journal of biological regulators and homeostatic agents. 32(6), 1433-41.

5. Masuko K, Murata M, Yudoh K, Kato T, Nakamura H. (2009) Anti-inflammatory effects of hyaluronan in arthritis therapy: effective treatment in patients affected by low degree GA, a finding in keeping with established data from literature $(3,4)$. In addition, we found that this therapeutic course produced a significant and perduring improvement also in the worse GA cases.

The features characterizing the therapeutic course studied herein likely depend on the rational timing of injections with LHA and CLHA, which might exploit and optimize the pharmacokinetic and pharmacodynamics properties of the two forms of HA, in such a way that this regimen results in affordable clinical efficacy and, ultimately, patients' satisfaction. Such an interpretation is in keeping with other and independent evidences indicating that the pharmacodynamic features of HA may profoundly vary as a function of size, structure and chemical modifications $(7,9,10,29)$. These results implicitly suggest that combining different forms of HA such as novel cooperative hybrid complexes or sequential/timed administration of linear and crosslinked preparations as in our case - instead of administering a single, specific form - could recruit multiple and converging mechanisms exalting the pleiotropic nature of native HA.

Today, different forms of HA are available, but there is still the need to identify the basis for their rationale combination, either within a single preparation, or over a multi-injection treatment schedule.

Hence - although the pharmacokinetic/pharmacodynamic issue of in syringe and/or over time combinations of different forms of HA was beyond the scope of the present study, based on our and other groups' independent results and considerations, it is advisable that future researches will be aimed at specifically deal with this point.

Not just for viscosity. International journal of general medicine. 2, 77-81.

6. Rayahin JE, Buhrman JS, Zhang Y, Koh TJ, Gemeinhart RA. (2015) High and low molecular weight hyaluronic acid differentially influence macrophage activation. ACS biomaterials science \& engineering. 1(7), 481-93.

7. Schiraldi C, Stellavato A, de Novellis F, La Gatta A, De Rosa M. (2016) Hyaluronan viscosupplementation: state of the art and insight into the novel cooperative hybrid complexes based on high and low molecular weight HA of potential interest in osteoarthritis treatment. Clinical cases in mineral and bone metabolism : the official journal of the Italian Society of Osteoporosis, Mineral Metabolism, and Skeletal Diseases. 13(1), 36-7.

8. Stellavato A, Vassallo V, La Gatta A, Pirozzi AVA, De Rosa M, Balato G, et al. (2019) Novel Hybrid Gels Made of High and Low Molecular Weight Hyaluronic Acid Induce Proliferation and Reduce Inflammation in an Osteoarthritis In Vitro Model Based on Human Synoviocytes and Chondrocytes. BioMed research international. 2019, 4328219. 
9. Tuan S, Liou I, Su H, Tsai Y, Chen G, Sun S. (2018) Improvement of self-reported functional scores and thickening of quadriceps and femoral intercondylar cartilage under ultrasonography after single intra-articular injection of a novel cross-linked hyaluronic acid in the treatment of knee osteoarthritis. Journal of back and musculoskeletal rehabilitation. 31(4), 709-18.

10. Oliviero F, Scanu A, Ramonda R, Frallonardo P, Sfriso P, Dayer JM, et al. (2015) IL-1ss and IL-8 are scavenged by the hexadecylamide derivative of hyaluronic acid: a new mechanism. Journal of biomedical materials research Part A. 103(9), 2823-9.

11. Bellamy N, Campbell J, Welch V, Gee TL, Bourne R, Wells GA. (2006) Intraarticular corticosteroid for treatment of osteoarthritis of the knee. Cochrane Database of Systematic Reviews. (2).

12. Moskowitz RW. (2007) Osteoarthritis : diagnosis and medical/ surgical management. pp. xviii, 470 p. Wolters Kluwer Health/ Lippincott Williams \& Wilkins, Philadelphia.

13. Snelling SJB, Bas S, Puskas GJ, Dakin SG, Suva D, Finckh A, et al. (2017) Presence of IL-17 in synovial fluid identifies a potential inflammatory osteoarthritic phenotype. PloS one. 12(4), e0175109-e.

14. Padulo J, Oliva F, Frizziero A, Maffulli N. (2016) Muscles, Ligaments and Tendons Journal - Basic principles and recommendations in clinical and field Science Research: 2016 Update. Muscles, ligaments and tendons journal. 6(1), 1-5.

15. Grimby G, Borjesson M, Jonsdottir IH, Schnohr P, Thelle DS, Saltin B. (2015) The "Saltin-Grimby Physical Activity Level Scale" and its application to health research. Scandinavian journal of medicine \& science in sports. 25 Suppl 4, 119-25.

16. Ucar D, Diracoglu D, Suleyman T, Capan N. (2013) Intra-articular hyaluronic Acid as treatment in elderly and middleaged patients with knee osteoarthritis. The open rheumatology journal. $7,38-41$.

17. Lenssen AF, van Dam EM, Crijns YHF, Verhey M, Geesink RJT, van den Brandt PA, et al. (2007) Reproducibility of goniometric measurement of the knee in the in-hospital phase following total knee arthroplasty. BMC musculoskeletal disorders. 8,83 .

18. Bellamy N, Wilson C, Hendrikz J. (2011) Population-Based Normative Values for the Western Ontario and McMaster (WOMAC) Osteoarthritis Index: Part I. Seminars in Arthritis and Rheumatism. 41(2), 139-48.

19. Abate M, Vanni D, Pantalone A, Salini V. (2017) Hyaluronic acid in knee osteoarthritis: preliminary results using a four months administration schedule. International Journal of Rheumatic Diseases. 20(2), 199-202.

20. Saarakkala S, Waris P, Waris V, Tarkiainen I, Karvanen E, Aarnio J, et al. (2012) Diagnostic performance of knee ultrasonography for detecting degenerative changes of articular cartilage. Osteoarthritis and Cartilage. 20(5), 376-81.

21. Sestili P, Barbieri E, Martinelli C, Battistelli M, Guescini M, Vallorani L, et al. (2009) Creatine supplementation prevents the inhibition of myogenic differentiation in oxidative- ly injured C2C12 murine myoblasts. Molecular Nutrition \& Food Research. 53(9), 1187-204.

22. Frizziero A, Vittadini F, Oliva F, Abatangelo G, Bacciu S, Berardi A, et al. (2018) I.S.Mu.L.T. Hyaluronic acid injections in musculoskeletal disorders guidelines. Mltj-Muscle Ligament. 8(3), 364-98.

23. Podlipska J, Guermazi A, Lehenkari P, Niinimaki J, Roemer FW, Arokoski JP, et al. (2016) Comparison of Diagnostic Performance of Semi-Quantitative Knee Ultrasound and Knee Radiography with MRI: Oulu Knee Osteoarthritis Study. Scientific reports. 6, 22365.

24. Schmitz RJ, Wang HM, Polprasert DR, Kraft RA, Pietrosimone BG. (2017) Evaluation of knee cartilage thickness: A comparison between ultrasound and magnetic resonance imaging methods. The Knee. 24(2), 217-23.

25. Pradsgaard DO, Fiirgaard B, Spannow AH, Heuck C, Herlin T. (2015) Cartilage thickness of the knee joint in juvenile idiopathic arthritis: comparative assessment by ultrasonography and magnetic resonance imaging. J Rheumatol. 42(3), 534-40.

26. Spannow AH, Stenboeg E, Pfeiffer-Jensen M, Fiirgaard B, Haislund M, Ostergaard M, et al. (2011) Ultrasound and MRI measurements of joint cartilage in healthy children: a validation study. Ultraschall in der Medizin. 32 Suppl 1, S110-6.

27. Bruyere O, Collette J, Kothari M, Zaim S, White D, Genant H, et al. (2006) Osteoarthritis, magnetic resonance imaging, and biochemical markers: a one year prospective study. Annals of the rheumatic diseases. 65(8), 1050-4.

28. Kundu-Raychaudhuri S, Abria C, Raychaudhuri SP. (2016) IL-9, a local growth factor for synovial T cells in inflammatory arthritis. Cytokine. 79, 45-51.

29. Oliviero F, Sfriso P, Baldo G, Dayer J, Giunco S, Scanu A, et al. (2009) Apolipoprotein AI and cholesterol in synovial fluid of patients with rheumatoid arthritis, psoriatic arthritis and osteoarthritis. Clinical \& Experimental Rheumatology. 27(1), 79.

30. Chang X, Cui Y, Zong M, Zhao Y, Yan X, Chen Y, et al. (2009) Identification of proteins with increased expression in rheumatoid arthritis synovial tissues. The Journal of Rheumatology. jrheum. 080939.

31. Olszewska-Slonina D, Matewski D, Jung S, Olszewski KJ, Czajkowski R, Braszkiewicz J, et al. (2013) The activity of cathepsin D and alpha-1 antitrypsin in hip and knee osteoarthritis. Acta Biochimica Polonica. 60(1).

32. Akasaki Y, Reixach N, Matsuzaki T, Alvarez-Garcia O, Olmer M, Iwamoto Y, et al. (2015) Transthyretin deposition in articular cartilage: a novel mechanism in the pathogenesis of osteoarthritis. Arthritis \& Rheumatology. 67(8), 2097-107.

33. Yoshida M, Sai S, Marumo K, Tanaka T, Itano N, Kimata K, et al. (2004) Expression analysis of three isoforms of hyaluronan synthase and hyaluronidase in the synovium of knees in osteoarthritis and rheumatoid arthritis by quantitative realtime reverse transcriptase polymerase chain reaction. Arthritis research \& therapy. 6(6), R514-20. 